\title{
Perfil de utilização de fitoterápicos em farmácias comunitárias de Belo Horizonte sob a influência da legislação nacional
}

\author{
A.Q. Ribeiro ${ }^{1,2 *}$, J.P.V. Leite ${ }^{2}$,A.M. Dantas-Barros ${ }^{3}$ \\ ${ }^{I}$ Centro de Estudo do Medicamento, Departamento de Farmácia Social, Faculdade de Farmácia, UFMG, Avenida \\ Antônio Carlos 6627, Pampulha, 31270-901, Belo Horizonte, MG, Brasil, \\ ${ }^{2}$ Faculdade de Ciências Biológicas e da Saúde, Centro Universitário Newton Paiva, Av. Silva Lobo 1730, Nova \\ Granada, 30100-000, Belo Horizonte, MG, Brasil, \\ ${ }^{3}$ Departamento de Produtos Farmacêuticos, Faculdade de Farmácia, UFMG, Av. Antônio Carlos 6627, Pampulha, \\ 31270-901, Belo Horizonte, MG, Brasil
}

\begin{abstract}
RESUMO: Um panorama do consumo de fitoterápicos em farmácias comunitárias de Belo Horizonte $(\mathrm{MG})$ é utilizado neste artigo como instrumento para analisar a influência da legislação nacional sobre este mercado. Para tal, foram entrevistados indivíduos que adquiriram fitoterápicos nos estabelecimentos farmacêuticos visitados. Observa-se que, na mesma nação detentora da maior biodiversidade do planeta e privilegiada por uma cultura popular conhecedora das propriedades terapêuticas das plantas, existe uma tendência de mercado para a utilização de plantas exóticas, oriundas de países desenvolvidos. A influência da legislação brasileira de fitoterápicos, que prima pela qualidade, eficácia e segurança desses medicamentos, mas que, ao mesmo tempo, tende a proscrever as drogas nativas que fazem parte da medicina popular brasileira, é discutida nesse trabalho. Verifica-se, assim, a pertinência de se discutir o perfil de usuário de fitoterápicos à luz das normas de registro desses fármacos no país no campo da saúde pública, tendo em vista que essa legislação repercute nas estratégias e/ou empecilhos para aumentar o acesso a medicamentos pela população.
\end{abstract}

Unitermos: Fitoterápicos, drogas vegetais, acesso a medicamentos, legislação.

\begin{abstract}
Profile of the utilization of phytotherapie medicines in communitary pharmacies of Belo Horizonte, under the influence of the national legislation". An overview of the herbal drugs consumption in communitarian pharmacies in Belo Horizonte (MG), Brazil is used in this article as a tool to analyze the influence of the national law over this market. In order to reach this goal, individuals that had bought herbal drugs were interviewed at the pharmacies. It was observed that, in the same nation that has the highest biodiversity in the planet and is privileged by a popular culture knowledgeable in the therapeutic properties of the plants, there is a tendency in the market for the use of exotic plants, coming from developed countries. The influence of the Brazilian law about the herbal drugs, which constantly worries about the quality, efficiency and safety of these medicines, but at the same time, tends to proscribe the native drugs that are part of the popular medicine, is discussed in this assignment. It is concluded that it is pertinent to discuss the profile of the herbal drugs users in the spot of the laws of the country related to these drugs, in the field of the public health, since this legislation is reflected in the strategies and/or barriers to increase the access to the drugs by the population.
\end{abstract}

Keywords: Herbal drugs, vegetal drugs, drug access, law.

\section{INTRODUÇÃO}

Atualmente, observa-se um crescimento na utilização de fitoterápicos pela população brasileira. Alguns fatores poderiam explicar o aumento do uso desses medicamentos, como os avanços ocorridos na área científica que permitiram o desenvolvimento de fitoterápicos reconhecidamente seguros e eficazes, como também uma forte tendência de busca, pela população, por terapias menos agressivas destinadas ao atendimento primário à saúde (Yunes; Pedrosa; Cechinel Filho,
2001).

De acordo com a legislação em vigor no país, entende-se como fitoterápico "aquele medicamento obtido empregando-se exclusivamente matérias-primas vegetais. É caracterizado pelo conhecimento da eficácia e dos riscos do seu uso, assim como pela reprodutibilidade e constância de sua qualidade. Sua eficácia e segurança são validadas através de levantamentos etnofarmacológicos de utilização, documentações tecnocientíficas em publicações ou ensaios clínicos fase 3" (Brasil, 2004a).

A partir da publicação em fevereiro de $2000 \mathrm{da}$ 


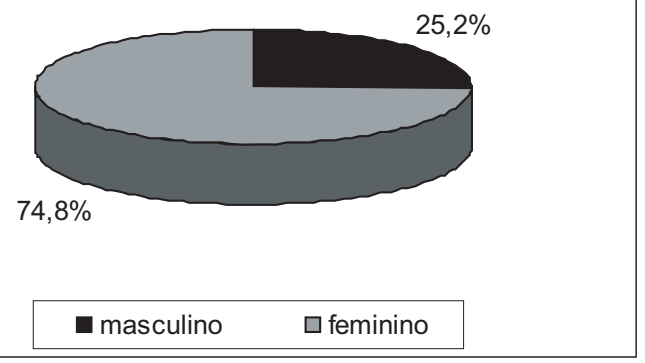

Figura 1. Perfil dos indivíduos que adquiriram fitoterápicos nos estabelecimentos farmacêuticos de acordo com o sexo, no período do estudo, Belo Horizonte (MG), 2003

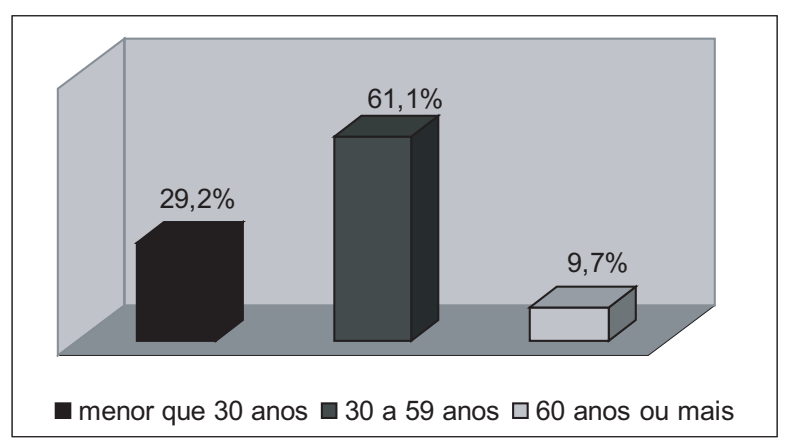

Figura 2. Perfil dos indivíduos que adquiriram fitoterápicos nos estabelecimentos farmacêuticos de acordo com a idade, no período do estudo, Belo Horizonte (MG), 2003.

RDC 17 (Brasil, 2000), a indústria farmacêutica passou a ter novas normas para o registro de fitoterápicos no Brasil. Desde então, o mercado nacional teve que se adequar às novas regras, de forma a garantir a qualidade, eficácia e segurança destes medicamentos. Em março de 2004, esta Resolução foi revogada pela publicação de uma nova Resolução, a RDC 48 (Brasil, 2004a). Apesar da RDC 48 trazer algumas mudanças conceituais em relação à legislação anterior, na tentativa de se adequar a outras normas de medicamentos da Agência Nacional de Vigilância Sanitária (ANVISA), o critério de registro para fitoterápicos não sofreu grandes alterações.

A ANVISA reconhece, dentro daqueles fitoterápicos com maior número de estudos científicos, uma lista de drogas vegetais que têm a permissão de obter o registro simplificado pela indústria (Brasil, 2004b), não havendo necessidade de validar as indicações terapêuticas e segurança de uso. Desta lista, contendo 34 espécies vegetais, a maioria trata-se de plantas exóticas, ou seja, aquelas que não medram espontaneamente no Brasil, sendo originárias de outros países, sobretudo da Europa e América do Norte, onde as espécies vegetais locais são alvo de maior número de investigação científica.

Caso o fitoterápico não integre a "Lista de registro simplificado de fitoterápicos", cabe à indústria farmacêutica, para o lançamento de um fitoterápico no mercado nacional, dentre outras exigências, comprovar a segurança e eficácia do produto. Para esta comprovação é possível seguir três diferentes caminhos. A primeira opção, que por ser a maior onerosa é a menos utilizada pela indústria, consiste na realização de testes de segurança (toxicologia pré-clínica e clínica) e de eficácia terapêutica (farmacologia pré-clínica e clínica) do medicamento. Vale ressaltar que, nos últimos anos, esta opção fez com que houvesse uma aproximação das empresas com os centros de pesquisas brasileiros, possibilitando a realização de parcerias para a execução de alguns desses estudos (Alves, 2004).

Como segunda opção para se garantir a segurança e eficácia do fitoterápico, a legislação permite que a indústria farmacêutica se utilize da apresentação de monografias da droga vegetal presente na formulação do medicamento, que atestem sobre estes estudos. Para se certificar da seriedade dessas monografias, a ANVISA publicou uma "Lista de referências bibliográficas para avaliação de segurança e eficácia de medicamentos fitoterápicos" (Brasil, 2004c), colocando as referências dentro de um elenco de pontuação, de acordo com a "qualidade científica" da obra. Dessa forma, para o laboratório farmacêutico comprovar a segurança e eficácia do fitoterápico por meio de apresentação de estudos descritos nessas obras, o produto deve atingir, no mínimo, seis pontos, conferidos de acordo com a escala de pontuação das referências. Nesse caso, grande 
parte das obras refere-se a compêndios internacionais que contemplam estudos realizados principalmente com plantas oriundas de países europeus.

A terceira opção trata-se do laboratório apresentar um levantamento etnofarmacológico ou de documentações tecnocientíficas que avaliem a indicação de uso, a coerência com relação às indicações terapêuticas propostas, ausência de risco tóxico ao usuário e comprovação de uso seguro por um período igual ou superior a 20 anos (Brasil, 2004a).

Dessa forma, se por um lado a legislação consegue reforçar o critério de segurança e eficácia dos fitoterápicos registrados no Brasil, ela também privilegia de certa forma a incorporação, no nosso mercado farmacêutico, daquelas plantas consideradas exóticas, uma vez que a maioria das obras na literatura colocada como referência não contempla estudos clínicos de plantas da flora brasileira utilizadas na medicina popular. Vale ressaltar que os resultados de segurança e eficácia clínica aceitos pela legislação para o registro dessas drogas vegetais foram obtidos em estudos realizados com população de perfil epidemiológico diferente da brasileira, colocando em questão a necessidade de cautela na transposição desses resultados para nossa população.

Outro agravante dos critérios utilizados pela legislação brasileira de fitoterápicos diz respeito à desvalorização da medicina popular, uma vez que a maior parte das plantas utilizadas tradicionalmente pela população ainda não foi avaliada do ponto de vista toxicológico e farmacológico, o que tornaria proibitivo o seu registro como fitoterápico. $\mathrm{O}$ conhecimento intuitivo das propriedades dessas plantas é resultado da riqueza da sócio-biodiversidade brasileira, que por transferência entre diferentes gerações foi-se constituindo como um importante arsenal terapêutico (Elisabetsky; Shanley, 1994). Na pesquisa com plantas medicinais já é devidamente reconhecida a importância da dialética entre os conhecimentos racionais (científicos) e aqueles empíricos (intuitivos), como é demonstrado em diversos trabalhos de etnofarmacologia (Elisabetsky; Posey, 1986). O complemento dessas duas formas de saber fazse de fundamental importância para o aproveitamento terapêutico racional dos nossos recursos vegetais. Se por um lado encontra-se no país importante levantamento sobre o uso de plantas na medicina popular (Lorenzi; Matos, 2002), por outro ainda faltam estudos científicos que comprovem a utilização segura e eficaz de várias dessas plantas.

Existe também um arsenal de informações resultantes de pesquisa da flora brasileira que nem sempre é compilado de forma que possa servir como subsídio para a inclusão na "Lista de referência bibliográfica para avaliação de segurança e eficácia de medicamentos fitoterápicos", disponibilizado pela ANVISA. Para o melhor aproveitamento desses dados no país seria necessária a realização de um grande programa de catalogação, análise e síntese dos resultados, de forma a construir um memento fitoterápico com informações confiáveis.

Assim, buscando analisar a influência da legislação que normatiza o registro de medicamentos fitoterápicos no Brasil sob o mercado farmacêutico nacional, o presente trabalho objetivou identificar o perfil de utilização de fitoterápicos em farmácias comunitárias de Belo Horizonte (MG) e a influência da legislação sobre esta realidade.

\section{MATERIAL E MÉTODOS}

O presente estudo foi realizado no primeiro semestre de 2003 e integra o Projeto Assistência Farmacêutica e Fitoterapia, desenvolvido pelo Centro de Estudo do Medicamento (Cemed) em parceria com o Departamento de Produtos Farmacêuticos, ambos da Faculdade de Farmácia da UFMG. Para a realização do estudo, foi obtida uma amostra aleatória de 10,0\% dos estabelecimentos farmacêuticos (com exceção das farmácias de manipulação) de Belo Horizonte, o que correspondeu a 102 estabelecimentos. Estes estabelecimentos foram visitados pelos alunos da disciplina de Farmacognosia do Curso de Farmácia da UFMG, os quais foram responsáveis pela coleta dos dados.

Acoleta dos dados foi feita por meio de entrevistas diretas com indivíduos que adquiriram fitoterápicos nos estabelecimentos farmacêuticos visitados. Os alunos foram orientados a entrevistar o primeiro indivíduo que adquirisse pelo menos um fitoterápico para uso próprio no momento da visita ao estabelecimento sorteado. Em caso de recusa, o aluno entrevistaria o próximo indivíduo que atendesse à condição mencionada e concordasse em participar da pesquisa. Logo, em cada estabelecimento farmacêutico sorteado foi entrevistado um indivíduo. Para a coleta dos dados foi utilizado um questionário estruturado, com perguntas fechadas e abertas, o qual foi testado em fase piloto. Este questionário continha variáveis relacionadas ao indivíduo, tais como sexo, idade e escolaridade e variáveis relacionadas ao fitoterápico, tais como nome do produto adquirido e se este era associação de drogas vegetais.

Os dados obtidos foram armazenados em um banco de dados no Programa Excel e analisados com o auxílio do software Epi-Info 2000. A análise quantitativa dos dados constou de distribuição de freqüências, cálculos de médias, medianas e desvios-padrão. Numa análise posterior, os dados levantados foram confrontados com os critérios para o registro de fitoterápicos estabelecidos pela legislação brasileira.

\section{RESULTADOS}

No período de junho a julho de 2003 foram entrevistados 102 indivíduos nos estabelecimentos farmacêuticos estudados em Belo Horizonte. Os 
Tabela 1. Perfil dos indivíduos que adquiriram fitoterápicos nos estabelecimentos farmacêuticos de acordo com a escolaridade, no período do estudo, Belo Horizonte (MG), 2003.

\begin{tabular}{lcc}
\hline \multicolumn{1}{c}{ ESCOLARIDADE } & FREQUENCIA & PERCENTUAL \\
& $\mathrm{n}$ & $\%$ \\
\hline Curso superior & 26 & 25,5 \\
Segundo grau & 53 & 52,0 \\
Primeiro grau & 16 & 15,7 \\
Curso primário & 3 & 2,9 \\
Curso primário incompleto & 1 & 1,0 \\
Outros & 3 & 2,9 \\
\hline TOTAL & 102 & 100,0 \\
\hline
\end{tabular}

Tabela 2. Distribuição dos fitoterápicos mais adquiridos pelos entrevistados, de acordo com a droga vegetal, no período do estudo, Belo Horizonte (MG), 2003.

\begin{tabular}{lcc}
\hline \multicolumn{1}{c}{ DROGA VEGETAL } & FREQUENNCIA & PERCENTUAL \\
& $\mathrm{n}$ & 16,3 \\
\hline 1. Ginkgo biloba & 21 & 10,8 \\
2. Passiflora spp. & 14 & 10,1 \\
3. Aesculus hippocastanum & 13 & 9,3 \\
4. Cassia spp. & 12 & 6,2 \\
5. Paullinia cupana & 8 & 4,6 \\
6. Hipericum perforatum & 6 & 3,9 \\
7. Rhamnus purshiana & 5 & 3,9 \\
8. Crataegus oxyacantha & 5 & 3,9 \\
9. Salix alba & 5 & 3,9 \\
10. Valeriana officinalis & 5 & 3,1 \\
11. Allium sativum & 4 & 24,0 \\
12. Outras & 31 & 100,0 \\
\hline TOTAL & 129 & \\
\hline
\end{tabular}

resultados mostram que a maioria era do sexo feminino, correspondendo a quase $75,0 \%$ dos entrevistados (Figura 1).

Em relação à idade dos indivíduos, esta variou de 18 a 84 anos, com uma média de 41,9 anos ( $\pm 16,1$ anos) e mediana de 43 anos. A maioria dos entrevistados possuía entre 30 e 59 anos, o que equivale a $61,1 \%$ do total (Figura 2).

No que se refere à escolaridade dos entrevistados, foi observado que a maior parcela $(48,7 \%)$ possuía segundo grau completo, como mostra a Tabela 1.

A análise considerando os fitoterápicos adquiridos mostra que a maioria (91) era representada por monodrogas, ou seja, a formulação do produto fitoterápico apresentava apenas uma droga vegetal, o que corresponde a $89,0 \%$ dos produtos.

$\mathrm{O}$ total de 102 fitoterápicos adquiridos corresponde a 129 drogas vegetais. Assim, ao se estratificar os fitoterápicos mais adquiridos de acordo com a droga vegetal, observou-se que a Gingko biloba foi a droga vegetal mais procurada, respondendo por $23,4 \%$ das procuras. Em seguida, aparece a Passiflora spp. e a droga vegetal Aesculus hippocastanum (Tabela 2).

Dentre as dez drogas vegetais mais adquiridas nos estabelecimentos pesquisados, sete referem-se a espécies vegetais exóticas, que não medram espontaneamente no Brasil e que não fazem parte da medicina popular brasileira. Assim, observa-se que dentre estas, apenas as espécies Passiflora spp., Cassia spp. e Paullinia cupana são nativas ou domesticadas no Brasil, sendo que as demais necessitam ser importadas dos seus países de origem pelas indústrias brasileiras para a produção de fitoterápicos ou, como em alguns casos, realizar a importação do medicamento fitoterápico acabado.

\section{DISCUSSÃO}

De acordo com os resultados obtidos, a grande maioria dos indivíduos que adquiriram fitoterápicos no período do estudo era representada por mulheres entre 30 e 59 anos. O predomínio do sexo feminino entre os indivíduos que utilizam medicamentos convencionais já tem sido demonstrado em diferentes estudos nacionais e internacionais (Bertoldi et al., 2004; Metge et al., 1999). Como são escassos os estudos que abordem esta questão no contexto da medicina complementar no Brasil, objetivamos explorá-la. Assim, nossos resultados sugerem uma maior aceitabilidade dessa terapia pelas mulheres. 
Em que se pesem as diferenças metodológicas, este fato também é observado em países desenvolvidos (Yu; Ghandour; Huang, 2004; Kaufman et al., 2002). Uma vez que várias são as razões para este perfil, o maior interesse nesta questão refere-se à maior propensão das mulheres para receberem prescrições médicas como para se automedicarem, fato este que as tornam mais vulneráveis aos prejuízos oriundos do uso de muitos medicamentos, como um maior risco de reações adversas.

Mais da metade dos usuários possui escolaridade igual ou superior ao nível secundário. Este achado é bastante interessante, pois sugere que a maior escolaridade seja um determinante do uso de fitoterápicos em farmácias comunitárias. Estudos no âmbito internacional têm evidenciado que entre as principais características de usuários de fitoterápicos e outras terapias complementares são as de possuírem maiores níveis de escolaridade (Yu; Ghandour; Huang, 2004; Astin, 1998). Uma vez que já se conhece uma relação direta entre escolaridade e renda, é possível especular que, na amostra estudada por nós, os usuários de fitoterápicos também possuam maior poder aquisitivo. Esse fato aponta para a necessidade de aprofundamento no estudo desta relação bem como no estudo do custo dos tratamentos com fitoterápicos. À luz da legislação vigente, é pertinente discutir se as normas de registro de fitoterápicos no país constituem estratégias ou empecilhos para aumentar o acesso a medicamentos, tendo em vista que este é um problema de saúde pública.

Pelos resultados da pesquisa, observou-se também um elevado percentual de fitoterápicos adquiridos como monodrogas. Este fato pode ser explicado pela maior exigência para o registro daqueles fitoterápicos que contemplam duas ou mais drogas vegetais, sendo necessária a realização de pesquisa científica que mostre a eficiência e segurança da associação, já que a literatura científica disponibiliza as referências de estudos realizados com as drogas vegetais individuais.

Como foi visto, dentre as dez drogas vegetais mais adquiridas, observa-se que apenas as espécies Passiflora spp., Cassia spp. e Paullinia cupana são nativas ou domesticadas no Brasil, sendo as demais consideradas exóticas, tendo que as indústrias brasileiras importarem dos seus países de origem. Esse contexto faz com que no campo de medicamentos fitoterápicos no país se repita a situação que ocorre na área de medicamentos sintéticos, onde a grande parte da matéria-prima é importada, não sendo produzida no Brasil. Esse cenário do mercado nacional de fitoterápico, onde na cadeia produtiva do medicamento se exclui a obtenção de drogas vegetais em solo brasileiro, retira também do seu contexto o produtor rural, levando em conta que a proscrição das drogas vegetais nativas deixa de envolver esse ator social que poderia estar agregando valores à agricultura nacional, sobretudo aquela familiar, já que no Brasil as pequenas propriedades rurais representam os principais locais de produção de ervas medicinais (Silva et al., 2001).

Pode-se também verificar que três drogas vegetais - Ginkgo biloba, Passiflora spp. e Aesculus hippocastanum - respondem por mais da metade dos fitoterápicos adquiridos nas farmácias. Entre elas, destacase a Ginkgo biloba, cuja utilização tem sido difundida em vários países do mundo (Rigney; Kimber; Hindmarch, 1999). Vários estudos clínicos têm demonstrado que o extrato de Ginkgo biloba pode ser utilizado como agente nootrópico eficaz no tratamento sintomático de deficiências cognitivas (Schulz; Hansel; Tyler, 2002). Para as espécies do gênero Passiflora diversos estudos comprovaram os efeitos sedativo e ansiolítico de seus extratos (Della Loggia; Tubaro; Redaelli, 1981), enquanto para a espécie Aesculus hippocastanum diferentes pesquisas clínicas realizadas com extratos de suas sementes têm demonstrado sua atividade antiedema venoso em pacientes com insuficiência venosa crônica (Diehm et al., 1996). O grande número de estudos científicos realizados com essas drogas vegetais explica o fato delas estarem estarem entre aquelas mais utilizadas no mercado fitoterápico brasileiro (Suzuki, 2002). Esse dado indica a importância de se realizar pesquisas científicas com as plantas nativas brasileiras e de seus resultados servirem como subsídio para o registro de fitoterápicos.

\section{REFERÊNCIAS}

Alves FNR 2004. Desafios para o desenvolvimento de fitomedicamentos no Brasil no contexto da indústria farmacêutica. Rio de Janeiro: 248p. Dissertação de Mestrado - Escola Nacional de Saúde Pública Sérgio Arouca, Fundação Oswaldo Cruz.

Astin JA 1998. Why patient use alternative medicine: results of a national study. J Am Med Assn 279: 1548-1553.

Bertoldi AD, Barros AJD, Hallal PC, Lima RC 2004. Utilização de medicamentos em adultos: prevalência e determinantes individuais. Rev Saúde Pública 38: 228-238.

Brasil 2000. Ministério da Saúde, Agência Nacional de Vigilância Sanitária. RDC n ${ }^{\circ} 17$ de 24 de fevereiro de 2000. Dispõe sobre o registro de medicamentos fitoterápicos. Diário Oficial, Brasília, 25 fev.

Brasil 2004a. Ministério da Saúde, Agência Nacional de Vigilância Sanitária. RDC n 48 de 16 de março de 2004. Dispõe sobre o registro de medicamentos fitoterápicos. Diário Oficial, Brasília, 18 mar.

Brasil 2004b. Ministério da Saúde, Agência Nacional de Vigilância Sanitária. RE $n^{\circ} 89$ de 16 de março de 2004. Determina a publicação da "Lista de registro simplificado de fitoterápicos”. Diário Oficial, Brasília, 18 mar.

Brasil 2004c. Ministério da Saúde, Agência Nacional de Vigilância Sanitária. RE $n^{\circ} 88$ de 16 de março de 2004. Determina a publicação da "Lista de referências bibliográficas para a avaliação de segurança e eficácia de fitoterápicos". Diário Oficial, Brasília, 18 mar.

Della Loggia R, Tubaro A, Redaelli C 1981. Valutazione dell attività sul S.N.C del topo di alcuni estratti vegetali e di una loro assoziazione. Riv Neurol 51: 297-310.

Diehm C, Trampisch HJ, Lange S, Schmidt C 1996. Comparison 
of leg compression stocking and oral horse-chesnut seed extract therapy in patients with cronic venous insufficiency. Lancet 374: 292-294.

Elisabetsky E, Posey DA 1986. Pesquisa etnofarmacológica e recursos naturais no trópico úmido: o caso dos índios Kaiapó do Brasil e suas implicações para a ciência médica. Anais do I Simpósio do Trópico Úmido. Embrapa, CPATU, Documentos 36: 85-94.

Elisabetsky E, Shanley P 1994. Ethnopharmacology in the Brazilian Amazon. Pharmacol Ther 64: 201-214.

Kaufman DW, Kelly JP, Rosenberg L, Anderson TE, Mitchell AA 2002. Recent patterns of medication use in the ambulatory adult population of the United States - the Slone Survey. J Am Med Assn 287: 337-344.

Lorenzi H, Matos FJA 2002. Plantas medicinais no Brasil: nativas e exóticas. Nova Odessa: Instituto Plantarum, $512 \mathrm{p}$.

Metge C, Black C, Peterson S, Kozyrskjy AL 1999. The population's use of pharmaceuticals. Med Care 37: S42-S59.

Rigney U, Kimber S, Hindmarch I 1999. The effects of acute doses of standardized Ginkgo biloba extract on memory and psychomotor performance in volunteers. Phytother Res 13: 408-415.

Schulz V, Hansel R, Tyler VE 2002. Fitoterapia racional. $1^{\mathrm{a}} \mathrm{ed}$. Barueri: Manole, p. 42-56.

Silva SR, Buitrón X, Oliveira LH, Martins MVM 2001. Plantas medicinais do Brasil: aspectos gerais sobre legislação e comércio. Ministério de Cooperação Econômica e Desenvolvimento da Alemanha \& IBAMA, [Relatório final].

Suzuki SF 2002. O mercado de medicamentos fitoterápicos no Brasil. In: Schulz V, Hansel R, Tyler VE Fitoterapia racional. $1^{\text {a }}$ ed. Barueri: Manole, p. 363-369.

Yu SM, Ghandour RM, Huang ZJ 2004. Herbal supplement use among US women, 2000. J Am Med Womens Assn 59: $17-24$.

Yunes RA, Pedrosa RC, Cechinel Filho V 2001. Fármacos e fitoterápicos: a necessidade do desenvolvimento da indústria de fitoterápicos e fitofármacos no Brasil. Quím Nova 24: 147-152. 